\title{
Echoes of things to come. Ultrasound in UK emergency medicine practice
}

\author{
Jane Brenchley, John P Sloan, Peter K Thompson
}

\begin{abstract}
Ultrasound is widely used in the US and continental Europe in the immediate assessment of patients after blunt abdominal trauma. There are also now other recognised "primary" indications for ultrasound in emergency medicine.

In this paper current evidence supporting the implementation and use of emergency ultrasound in these primary conditions and possible other indications are assessed. The issues surrounding introduction of the technology into the practice of emergency medicine in this country are considered. It is accepted that further debate is necessary but the establishment of a robust evidence base in the UK will help to clarify the place of ultrasound.

(F Accid Emerg Med 2000;17:170-175)
\end{abstract}

Keywords: ultrasound

Ultrasound is an accepted, powerful diagnostic tool and is also used as assistance to facilitate invasive procedures. It is used by cardiologists, urologists, obstetricians/gynaecologists, trauma surgeons, and emergency physicians as well as by radiologists worldwide. ${ }^{1}$ Some of the early work demonstrating the benefits of ultrasound in the early assessment of trauma patients was initiated in the UK. ${ }^{2}$ However, over the past decade much investigation into the potential of ultrasound has occurred in the USA. The UK has been lagging behind for a number of reasons:

- Radiologists are uneasy about nonradiologists performing the examinations.

- There is little appreciation of the role of the focused examination (ruling in pathology as opposed to ruling out pathology).

Accident and

Emergency

Department,

Pinderfields Hospital,

Aberford Road,

Wakefield WF1 4DG

J Brenchley

Leeds General Infirmary, Leeds J P Sloan

King's College Hospital, London

P K Thompson

Correspondence to: Dr Brenchley, Specialist Registrar (e-mail:

jbrench@globalnet.co.uk)

Accepted for publication 10 November 1999
The practice of emergency medicine the rest of the hospital to offering an accurate diagnostic service and the emergency physician has become an emergency diagnostician. This focuses management for many conditions and may reduce lengthy patient waits and acute inpatient stays by allowing more appropriate patient disposal and treatment. Bedside diagnostic ultrasound provides an excellent imaging modality that assists the doctor in clinical management. If a service is available during normal working hours should we not be able to provide it out of hours? The concept of the " 24 hour hospital" is becoming widespread and ensures that patients arriving at any time of day receive a comparable service. Practitioners of emergency medicine borrow many techniques from other specialties. Incorporation of ultrasound into the emergency department is an extension of this process but has often been fraught with misunderstanding. Ultrasound in emergency medicine in this country will act as a clinical decision support tool and not replace formal imaging. Immediate access to ultrasonography in the emergency department will decrease individual patient waiting times in the department and provide a more rapid turnaround time, resulting in less overcrowding to the benefit of the whole department. Machines have reduced in price and once purchased further material costs are low. Staffing costs in terms of training, etc have yet to be assessed, but indications from elsewhere are that these are low. The initial providers of the service will be trained through a combination of dedicated courses and cooperation with their local radiology departments. Suitably trained emergency department personnel will then provide further training in-house.

There are many clinical areas where ultrasound has been shown to be useful in the initial diagnosis of patients in the emergency department. The primary uses of ultrasound are to answer six specific questions:

- Is there blood in the peritoneal cavity?

- Is an abdominal aortic aneurysm present?

- Is there evidence of cholelithiasis?

- Is there evidence of obstructive uropathy?

- Is there evidence of a living intrauterine pregnancy?

- Is there a pericardial effusion? ${ }^{1}$

There are other uses of ultrasounddescribed as secondary - within the emergency department including foreign body localisation, assessment of deep venous thrombosis, musculoskeletal imaging, and assistance in performing procedures. However, in all situations emergency ultrasound remains a specific, goal directed, focused examination employed to answer a single question, rather than fully evaluating a specific system. Comprehensive 
imaging of systems remains the domain of radiologists and will not be reduced by the implementation of specific ultrasound within the emergency department. The fuller evaluation obtained from a radiology departmental ultrasound continues as a predominantly daytime service and as such is not usually vital to the immediate diagnostic question.

\section{Clinical applications of emergency ultrasonography \\ PRIMARY INDICATIONS}

Focused assessment with sonography for trauma (FAST)

Focused assessment with sonography for trauma has been widely evaluated. ${ }^{3}$ This allows a timely examination, takes less than five minutes, and can be performed during resuscitation. ${ }^{4}$ It is readily repeatable and noninvasive and has replaced diagnostic peritoneal lavage as the primary assessment of blunt abdominal trauma. It will not replace other radiological procedures, for example double contrast computed tomography, but will more effectively triage patients to theatre, further investigation, or observation.

We suggest a possible algorithm for the use of FAST (see fig 1). In this example ultrasound is an additional step performed after the primary survey during resuscitation. More than $90 \%$ of scans provide a definite result. However, some scans are indeterminate and require either repeat ultrasonography (by emergency department staff), further clinical review or the use of additional investigations, which includes the use of diagnostic peritoneal lavage (DPL).

FAST employs a limited 4-6 view scan of the abdomen and pericardium purely for the purpose of detecting free fluid.

The standard views are: (1) Morison's pouch, (2) pericardial, (3) perisplenic space,

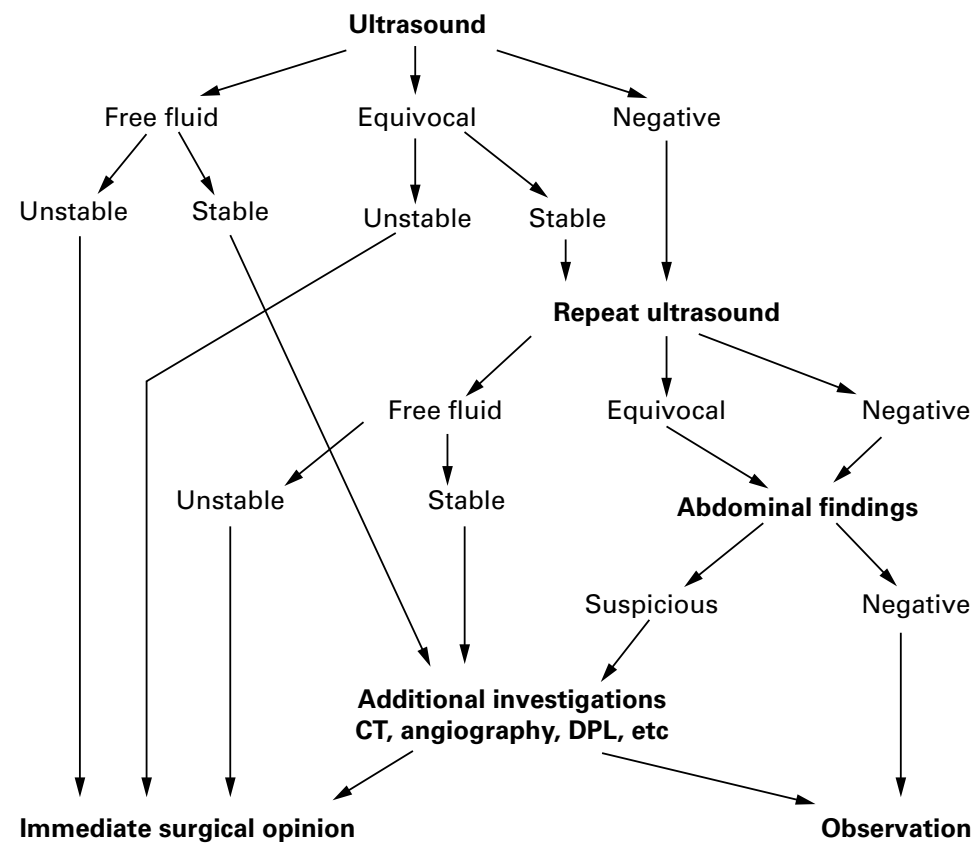

Figure 1 Algorithm for use of ultrasound in assessment of blunt abdominal trauma (CT = computed tomography; DPL = diagnostic peritoneal lavage).
(4) suprapubic, and (5) optional bilateral paracolic views. Of these, the most useful single view is of Morison's pouch, but adding other views increases sensitivity and specificity. ${ }^{67}$ Limitations of the technique include obesity, subcutaneous emphysema, and previous abdominal scars.

In a series of studies with FAST performed by surgeons, sensitivities ranged from $81.5 \%$ to 99\% (mean $90.1 \%$ ), specificity $95.0 \%-99.7 \%$ (mean $97.7 \%) .^{7-19}$ Initially pioneered in the US by trauma surgeons, there is increasing evidence that emergency physicians can perform the scan with similar sensitivity and specificity. Ma et al trained emergency physicians using 10 hours of instruction, then 15-20 examinations on normal subjects. The object of the training was to perform an examination within five minutes focusing on the identification of free fluid in the pericardial sac, the pleural cavity, the pararenal retroperitoneum, and the dependent intraperitoneal areas. They described subsequent emergency physician examination of 245 adult trauma patients and found $90 \%$ sensitivity, 99\% specificity, and 99\% accuracy compared with DPL, computed tomography, or laparotomy. ${ }^{20}$

There is as yet no consensus as to the minimum training required for performance of FAST. In one study, emergency medicine physicians were subjected to one hour of didactic teaching followed by one hour of practice on human volunteers. Sensitivity was $75 \%$, specificity $96 \%$, and accuracy $91 \%{ }^{6}$ The mean time for a scan in this study was $4.2 \pm 2.9$ minutes. However, recent studies have indicated a consistently steep learning curve and as few as 10 ultrasound scans may be required for clinician ultrasonographers to become competent in the FAST examination. ${ }^{8}$

\section{Abdominal pain and hypotension}

Although little reported, use of ultrasound to assess patients with hypotension and abdominal/flank pain is becoming standard practice. ${ }^{1}$ Aortic dimensions can be measured easily and although leakage cannot be assessed, the presence of a dilated aorta in patients with circulatory instability significantly speeds up diagnosis of a leaking abdominal aortic aneurysm and referral to the vascular team. The time to diagnosis of abdominal aortic aneurysm is consistently less than 10 minutes (D Plummer, personal communication, 1998). ${ }^{21}$ In these circumstances there is no attempt to define the proximal or distal limits of the aneurysm.

\section{Ectopic pregnancy}

Clinical assessment alone is inadequate in the management of symptomatic women in the first trimester and the early use of endovaginal ultrasound scanning is becoming the accepted standard of care..$^{22}$ In emergency medicine the goal of the examination is to identify a viable intrauterine pregnancy. ${ }^{23}$ Endovaginal scanning allows visualisation of intrauterine structures between one and two weeks earlier in gestation than transabdominal scans. ${ }^{24}$ Mateer et al evaluated the ability of emergency 
physicians to diagnose ectopic pregnancy. Six emergency physicians underwent 10-12 hours of didactic teaching followed by 10-12 patient examinations. A strict decision support protocol involving ultrasound appearances and serum human chorionic gonadotrophin concentration was adhered to. Altogether 118 examinations were performed over one year and a sensitivity of $99 \%$ and specificity of $93 \%$ was found. ${ }^{25}$

Shih measured the length of stay of patients in the emergency department. ${ }^{26}$ When ultrasound was performed by emergency physicians, there was a significant reduction on length of stay among patients with a viable intrauterine pregnancy (that is those who did not require further specialist consultation). Using a 24 hour teaching programme involving didactic and hands-on instruction and after proctoring of 10 studies in each of pelvic, aortic, and gall bladder imaging they achieved a sensitivity of $94 \%$ and specificity of $100 \%$ over 74 studies. A study involving endovaginal ultrasound performed by obstetric and gynaecology residents found emergency department stays were considerably shortened when ultrasound was performed in the emergency department. ${ }^{27}$ Their residents mastered the technique in a short time, although the precise length of training is not documented. Comparable results with ultrasonography performed by emergency physicians achieved a sensitivity of $90 \%$ and specificity of $88 \%$ over 125 patients. ${ }^{28}$ A recent study in Hong Kong using only transabdominal scans by emergency physicians showed a sensitivity of $80 \%$ and specificity of $78 \%$. Emergency department scans were compared with endovaginal scans performed by the gynaecologist. Agreement in diagnosis was $92 \%$ in the group referred to outpatients and only $65 \%$ in the group of patients admitted. ${ }^{29}$ These various studies indicate the clear benefit in the early use of both transabdominal and endovaginal ultrasonography in the expedient management of the complicated first trimester pregnancy.

\section{Echocardiography}

The two primary indications for emergency department echocardiography are the diagnosis of pericardial tamponade and the confirmation (or refutation) of pulseless electrical activity (electromechanical dissociation). ${ }^{1}$ A series of 1616 echocardiograms performed by emergency physicians to detect pericardial fluid had a positive and negative predictive value of 99.9\% (D Plummer, personal communication, 1998). As penetrating cardiac trauma becomes a more frequent occurrence ultrasound has been widely used to rapidly detect the presence of haemopericardium. Rozycki et al obtained pericardial scans by surgeons in 246 patients with penetrating wounds. ${ }^{30}$ The average scan time was 0.8 minutes and sensitivity, specificity, and accuracy were $100 \%$. A single subcostal view of the heart is included in the FAST protocol for the assessment of blunt trauma.

Ultrasound also allows the distinction between "true" electromechanical dissociation (EMD) and "clinical" EMD. True EMD is seen as organised electrical ventricular activity in the absence of visual evidence of myocardial contraction and carries a prognosis similar to asystole and stopping resuscitation is usually justified. Clinical EMD is diagnosed when myocardial contraction is visualised on ultrasonography and is usually associated with a potentially treatable cause. ${ }^{31}$ The correct identification of ventricular fibrillation masquerading as asystole has also been described and the use of ultrasound allows early defibrillation as part of successful resuscitation. ${ }^{32}$

\section{Renal colic}

Ultrasound is used in the assessment of patients with renal colic to detect hydronephrosis, and thus either complete or partial obstruction. ${ }^{33}$ The combination of ultrasound and a plain film of the kidneys, ureters, and bladder has been shown to have high sensitivity (97.1\%) but lower specificity (58.9\%) compared with intravenous pyelography. ${ }^{34}$ This is used to determine which patients need admission and those for whom outpatient follow up is more appropriate. The use of early renal ultrasonography by emergency physicians allows a progressive protocol for management of patients presenting with renal colic and so reduces the need for contrast imaging with its associated complications and inevitable time delays. ${ }^{1}$

\section{Gall stones}

Ultrasound is the primary diagnostic modality used to confirm the presence of gall stone disease and it has been shown that emergency physicians can produce accurate results. There is a high prevalence of gall stones within an aging population and the use of ultrasound assists in the assessment of patients presenting with abdominal pain. A combination of two or more of the following features is highly suggestive of acute gall bladder disease: the sonographic Murphy's sign (the point of maximal tenderness to transducer pressure is localised to the sonographically visualised gall bladder), a thickened gall bladder wall, gall bladder sludge, or pericholecystic fluid. ${ }^{121}$

\section{SECONDARY INDICATIONS}

Deep venous thrombosis (DVT)

The place of ultrasound in the diagnosis of DVT is well established but radiographic studies are only available during office hours, while patients attend emergency departments 24 hours a day. The role of emergency physicians in the management of patients presenting with possible DVT remains contentious; however, ultrasound provides an excellent modality for the effective identification of those who require treatment or further evaluation. Jolly et al investigated the use of colour Doppler ultrasonography by emergency physicians. ${ }^{35}$ Two physicians were trained by observing studies in the vascular laboratory and then performing 25-30 successful studies. A small number of patients were studied (23) but the emergency department examination was $100 \%$ sensitive and $75 \%$ specific with two false positives in patients who were subsequently found to have 
had a previous DVT. The successful use of ultrasound by emergency physicians was further confirmed in a recent study where emergency department ultrasounds were compared with vascular laboratory investigations (M Lambert, unpublished). In the two cases (total 112) where there was disagreement one was found to have been correctly diagnosed by the emergency department ultrasound when venography was performed and the other had been a false positive investigation. In an increasingly litigious environment the early use of ultrasonography to assess the likelihood of DVT is becoming the expected standard of care to allow early treatment.

\section{Musculoskeletal}

Within the UK there are units using ultrasound in the assessment of ligament injuries, epiphyseal and adolescent forearm fractures. ${ }^{36}$ However, it remains an underutilised resource and may provide an excellent further opportunity for rapid bedside diagnosis and consequent appropriate management of patients presenting to the emergency department.

OTHER APPLICATIONS

The quality of images provided through ultrasonography has made it the investigation of choice for foreign body localisation, ${ }^{37}{ }^{38}$ and the detection of soft tissue collections and abscesses. Its role in assisting with procedures through guidance (for example placement of central venous lines, ${ }^{39}{ }^{40}$ suprapubic aspiration, intercostal drain ${ }^{41}$ ) is also accepted. It is perhaps inevitable that many other applications will be explored, but a robust evidence base must be established before acceptance and universal practice is widespread. Within departments starting out on the use of ultrasound within their clinical practice it is suggested that they start with detection of free fluid and the gradual introduction of the other primary indications (D Plummer, personal communication, 1998). ${ }^{1}$

\section{Training}

One of the most controversial areas is the training required for emergency physicians in this country to practice ultrasound. The studies cited above show wide variation in the length of formal training and numbers of examinations. Even with brief training periods respectable sensitivities and specificities have been achieved. The Society for Academic Emergency Medicine has developed a model curriculum suggesting the adequate training of emergency physicians in the use of ultrasound. This recommends 40 hours of teaching and 150 examinations (at least $50 \%$ of these should be clinically indicated patient studies) across the range of indications. ${ }^{42}$ In the US $50 \%$ of emergency medicine residencies have formal ultrasound curricula with a further 30\% planning to implement one in the near future. ${ }^{44}$

Implementing the service in the UK is taking more time. However, the differences between healthcare systems across the Atlantic support a successful integration of emergency department ultrasound with the service provided by radiology. The guidelines from the Royal College of Radiologists for training of nonradiologists involve theoretical training in all aspects of the first FRCR examination syllabus that relate to ultrasound and practical training of 300 examinations in the first "module", and 150 examinations per module subsequently, supervised by radiologists. ${ }^{45}$ This has not been formulated with a view to the "rule in" use of ultrasound as performed by emergency physicians. Emergency physicians use ultrasound to document the presence of an abnormality (for example free fluid): to rule in pathology. Failure to visualise an abnormality on the scan is not used to "rule out" pathology until further investigations have been performed. Radiologists use ultrasound for both ruling in and ruling out pathology and this subtle but important difference does require a greater period of training. In the practice of emergency medicine ultrasound remains a clinical tool to assist in making a diagnosis. An equivocal or negative scan requires the doctor to exclude other potential causes or use different diagnostic techniques to reach a conclusion. Also, training through the radiology department is logistically difficult given the clinical throughput of most departments and the number of radiology trainees anxious to gain "hands-on" experience. ${ }^{46}$

It seems likely that emergency ultrasound, by focusing on single, goal directed issues, does not require the same in-depth knowledge of each system and thus shorter training periods are appropriate. The clinical opportunity available in most UK emergency departments allows sufficient throughput for emergency medicine specialist registrar training. Specific ultrasound courses provide an introduction to ultrasound and successfully teach the basics of ultrasound interpretation for the primary indications. The ongoing training can be provided by senior emergency physicians who develop their skills through departmental use and individual hospital arrangements with radiology colleagues. ${ }^{47}$

Concerns exist regarding skill maintenance and retention once trained. This has not been adequately investigated and remains an area for further evaluation. However, studies indicating the steep learning curve of nonradiologists in performing FAST scans had a small cohort of patients (234 over four years) and concluded that as few as 10 scans may provide competence. Equally, in the paper by Jolly et al evaluating DVT assessment, 100\% sensitivity and $75 \%$ specificity was obtained despite the two examiners seeing only 23 patients in total over 13 months. ${ }^{35}$ These findings suggest that skill maintenance is reasonable but this does require formal evaluation. The combination of primary indications should mean that ultrasound is used regularly and routinely in patient assessment and even in smaller departments skill decay should be minimal.

\section{The way forward}

It is clear from published work that emergency physicians can reliably perform and interpret 
limited ultrasound scans in the emergency setting and this has been shown to have positive effects on patient care, both in time spent in the emergency department and in time to diagnosis and definitive care..$^{20}$ We need to define and evaluate the role of emergency ultrasound in this country, and while it is sensible to proceed with caution we must rise to the challenge offered.

Recent debate in the UK literature has concentrated on the importance of having a hospital perspective, ${ }^{48}$ while the radiologists accept that there is a role for limited ultrasound by emergency physicians. ${ }^{49}$ We need to maintain both a specialty and a hospital perspective in order to obtain the full benefits ultrasound has to offer emergency medicine. The requirement for carefully monitored ultrasound use within emergency departments is common sense. We first have to successfully introduce a new skill to the specialty before we are in a position to fully evaluate the patient benefits and improvements in service efficiency. There is likely to be considerable apprehension on the part of some of our radiological colleagues. However, a recent survey of radiology clinical directors indicated a significant level of support for carefully monitored and trained emergency department use. Such an audited programme limited initially to a number of primary indications should establish whether ultrasound does have a role in patient care in our departments. This new beginning of emergency ultrasound within this country would be assisted by national support. The Faculty of Accident and Emergency Medicine together with the British Association for Accident and Emergency Medicine should consider formulating a policy statement that would firmly establish ultrasound as a priority within the development of emergency medical care in this country over the next decade. A suggested position statement is included in appendix 1. (The American College of Emergency Physicians position statement on emergency department ultrasonography is included for reference in appendix 2.)

\section{Appendix 1}

Suggested policy statement for UK emergency medicine

1. The prompt use of limited and focused bedside ultrasound providing specific answers is appropriate for an emergency physician to perform.

2. Emergency physicians using limited bedside ultrasound need appropriate training and hands-on experience to perform and evaluate their findings.

3. The primary indications for focused ultrasound are the following: traumatic haemoperitoneum, confirmation of intrauterine pregnancy, abdominal aortic aneurysm, pericardial fluid, evaluation of renal and biliary tract disease.

4. The Faculty of Accident and Emergency Medicine will evaluate and support the developing role of limited ultrasound within emergency medicine.

\section{Appendix 2}

Use of ultrasound imaging by emergency

physicians (American College of Emergency

Physicians (ACEP) policy statement fune 1997)

Ultrasound imaging enhances the physician's ability to evaluate, diagnose, and treat emergency patients. As these are often time dependent studies in acutely ill or injured patients, the emergency physician is in an ideal position to use this technology. Focused ultrasound examinations provide immediate information and can answer specific questions about the patient's physical condition. Such bedside ultrasound imaging is within the scope of practice of emergency physicians.

Therefore ACEP endorses the following principles:

- Ultrasound examination, interpretation, and clinical correlation should be available in a timely manner 24 hours a day for emergency department patients.

- Emergency physicians providing emergency ultrasound services should possess appropriate training and hands-on experience to perform and interpret limited bedside ultrasound imaging.

- ACEP specifically supports the use of ultrasound imaging by emergency physicians for at least the following clinical indications: traumatic haemoperitoneum, abdominal aortic aneurysm, pericardial fluid, ectopic pregnancy, and evaluation of renal and biliary tract disease.

- ACEP encourages continued research in the area of ultrasound imaging and any other known or evolving bedside imaging techniques and modalities.

- Training in performing and interpreting ultrasound imaging studies should be included in emergency medicine residency curricula.

Contributors

The primary idea for the review came from Mr J P Sloan and Dr Jane Brenchley. Dr Brenchley performed the literature search and wrote the provisional draft. Mr Sloan provided useful advice and criticism. Dr Peter $\mathrm{K}$ Thompson provided information on implementation of ultrasound and further revisions.

Funding: none.

Conflicts of interest: none.

1 Heller M. Jehle D. Ultrasound in emergency medicine. Philadelphia: WB Saunders, 1995

2 Chambers JA, Pilbrow WJ. Ultrasound in abdominal trauma: an alternative to peritoneal lavage. Arch Emerg Med 1988;5:26-33.

3 FAST Consensus Conference Committee. Focused assessment with sonography for trauma (FAST). Results from an Internationa Consensus Conference. 7 Trauma 1999;46: 466-72.

4 Mandavia D. Focussed abdominal sonography for trauma. $\mathcal{F}$ Emerg Med 1998;16:371.

5 Boulanger BR, Brenneman FD, Kirkpatrick AW, et al. The indeterminate abdominal sonogram in multisystem blunt indeterminate abdominal sonogram

6 Ingeman JE, Plewa MC, Okasinski RE, et al. Emergency physician use of ultrasonography in blunt abdominal trauma. Acad Emerg Med 1996;3:931-7.

$7 \mathrm{Ma}$ OJ, Kefer MP, Mateer JR, et al. Evaluation of haemoperitoneum using a single- vs multiple-view ultrasonographic examination. Acad Emerg Med 1995;2:581-6.

8 Shackford SR, Rogers FB, Osler TM, et al. Focused abdominal sonography for trauma: the learning curve of nonradiologist clinicians in detecting haemoperitoneum. $\mathcal{F}$ Trauma 1999;46:553-64.

9 Rozycki GS, Ochsner MG, Schmidt JA, et al. A prospective study of surgeon-performed ultrasound as the primary adjuvant modality for injured patient assessment. F Trauma 1995;39:492-8.

10 Hoffman R, Nerlich M, Muggia-Sullam M, et al. Blunt abdominal trauma in cases of multiple trauma evaluated by Trauma 1992;32:452-8.
Trasonography: a prospc 
11 Porter RS, Nester BA, Dalsey WC, et al. Use of ultrasound to determine need for laparotomy in trauma patients. Ann to determine need for laparo

12 Glaser K, Tschmelitsch J, Klingler P, et al. Ultrasonography in the management of blunt abdominal and thoracic trauma. Arch Surg 1994;129:743-7.

13 Rozycki GS, Ochsner MG, Jaffin JH, et al. Prospective evaluation of surgeons' use of ultrasound in the evaluation of trauma patients. F Trauma 1993;34:516-27.

14 Rothlin MA, Naf R, Amgwerd M, et al. Ultrasound in blunt abdominal and thoracic trauma. F Trauma 1993;34:48895.

15 Tso P, Rodriguez A, Cooper C, et al. Sonography in blunt abdominal trauma: a preliminary progress report. $\mathcal{F}$ Trauma 1992;33:39-44.

16 Gruessner R, Mentges B, Duber C, et al. Sonography versus peritoneal lavage in blunt abdominal trauma. F Trauma 1989;29:242-4.

17 Yoshii H, Sato M, Yamamoto S, et al. Usefulness and limitation of ultrasonography in the initial evaluation of blunt tion of ultrasonography in the initial evalua

18 Forster R, Pillasch J, Zielke A, et al. Ultrasonography in blunt abdominal trauma: influence of the investigators' experience. 7 Trauma 1993;34:264-9.

19 Goletti O, Ghiselli G, Lippolis PV, et al. The role of ultrasonography in blunt abdominal trauma: results in 250 consecutive cases. F Trauma 1994;36:178-81.

20 Ma OJ, Mateer JR, Ogata M, et al. Prospective analysis of a rapid trauma ultrasound examination performed by emergency physicians. $\mathcal{F}$ Trauma 1995;38:879-85.

21 Ballard RB, Rozycki GS, Knudson MM, et al. The surgeon's use of ultrasound in the acute setting. Surg Clin North Am 1998;78:337-64.

22 Phelan MB, Valley VT, Mateer JR. Pelvic ultrasonography. Emerg Med Clin North Am 1997;15:789-824.

23 Dart RG, Kaplan B, Varaklis K. Predictive value of history and physical examination in patients with suspected and physical examination in patients with susp

24 Dart RG. Role of pelvic ultrasonography in evaluation of symptomatic first trimester pregnancy. Ann Emerg Med 1999;33:310-20.

25 Mateer JR, Aiman EJ, Brown MH, et al. Ultrasonographic examination by emergency physicians of patients at risk for ectopic pregnancy. Acad Emerg Med 1995;2:867-73.

26 Shih CHY. Effect of emergency physician performed pelvic sonography on length of stay in the emergency department. Ann Emerg Med 1997;29:348-52.

27 Timor-Tritsch I, Greenidge S, Admon D, et al. Emergency room use of transvaginal ultrasonography by obstetrics and gynaecology residents. Am F Obstet Gynecol 1992;166:86672 .

28 Durham B, Lane B, Burbridge L, et al. Pelvic ultrasound performed by emergency physicians for the detection of ectopic pregnancy in complicated first-trimester pregnancies. Ann Emerg Med 1997;29:338-47.

29 Wong TW, Lau CC, Yeung A, et al. Efficacy of transabdominal ultrasound examination in the diagnosis of early pregnancy complications in an emergency department. $f$ Accid Emerg Med 1998;15:155-8.

30 Rozycki GS, Feliciano DV, Schmidt JA, et al. The role of surgeon-performed ultrasound in patients with possible cardiac wounds. Ann Surg 1996;223:737-46.
31 Mayron R, Gaudio FE, Plummer D, et al. Echocardiography performed by emergency physicians: impact on diagnosis and therapy. Ann Emerg Med 1988;17:150-4

32 Amaya SC, Langsam A. Ultrasound detection of ventricular fibrillation disguised as asystole. Ann Emerg Med 1999;33: 344-6.

33 Brown DFM, Rosen CL, Wolfe RE. Renal ultrasonography. Emerg Med Clin North Am 1997;15:877-93.

34 Henderson SO, Hoffner RJ, Aragona JL, et al. Bedside emergency department ultrasonography plus radiography of the kidneys, ureters and bladder vs intravenous pyelography in the evaluation of suspected ureteral colic. Acad Emerg Med 1998;5:666-71.

35 Jolly BT, Massarin E, Pigman EC. Colour Doppler ultrasonography by emergency physicians for the diagnosis of acute deep venous thrombosis. Acad Emerg Med 1997;4: $129-32$

36 Stewart J, Boyd R. Ultrasound scans and MRI of skeletal injuries in children. The AE E Letter RSM Press 1996;6(3): 7.

37 Hill R, Conron R, Greissinger P, et al. Ultrasound for the detection of foreign bodies in human tissue. Ann Emerg Med 1997;29:353-6.

38 Schlager D. Ultrasound detection of foreign bodies and procedure guidance. Emerg Med Clin North Am 1997;15:895 912.

39 Hilty WM, Hudson PA, Levitt MA, et al. Real time ultrasound-guided femoral vein catheterisation during cardiopulmonary resuscitation. Ann Emerg Med 1997;29:3316.

40 Gualtieri E, Deppe SA, Sipperley ME, et al. Subclavian venous catheterisation: greater success rate for less experienced operators using ultrasound guidance. Crit Care Med 1995;23:692-7.

41 Sisley AC, Rozycki GS, Ballard RB, et al. Rapid detection of traumatic effusion using surgeon performed ultrasonography. F Trauma 1998;44:291-5.

42 Lanoix R. Credentialing issues in emergency ultrasonography. Emerg Med Clin North Am 1997;15:913-20.

43 Mateer J, Plummer D, Heller M, et al. Model curriculum for physician training in emergency ultrasonography. Ann Emerg Med 1994;23:95-102.

44 Cook T, Roepke T. Prevalence and structure of ultrasound curricula in emergency medicine residencies. 7 Emerg Med 1998;16:655-7.

45 Board of the Faculty of Clinical Radiology, Royal College of Radiologists. Guidance for the training in ultrasound of medical non-radiologists. London: Royal College of Radiologists, 1997

46 Martin DF. Ultrasound for gastroenterologists. Gut 1996; 38:479-80.

47 Tandy TK, Hoffenberg S. Emergency department ultrasound services by emergency physicians: model for gaining hospital approval. Ann Emerg Med 1997;29:367-74.

48 Robinson N, Clancy M. Should UK emergency physicians undertake diagnostic ultrasound examinations? $\mathcal{F}$ Accid Emerg Med 1999;16:248-50.

49 Burnett $\mathrm{H}$, Nicholson D. Current and future role of ultrasound in the emergency department. $\mathcal{F}$ Accid Emerg Med 1999;16:250-4. 